\title{
Estimating the direct Covid-19 disability- adjusted life years impact on the Malta population for the first full year
}

\author{
Sarah Cuschieri ${ }^{1 *}$, Neville Calleja ${ }^{2,3}$, Brecht Devleesschauwer ${ }^{4,5}$ and Grant M. A. Wyper ${ }^{6}$
}

\begin{abstract}
Background: Disability-adjusted life years (DALYs) combine the impact of morbidity and mortality, allowing for comprehensive comparisons of the population. The aim was to estimate the DALYs due to Covid-19 in Malta (March 2020-21) and investigate its impact in relation to other causes of disease at a population level.

Methods: Mortality and weekly hospital admission data were used to calculate DALYs, based on the European Burden of Disease Network consensus Covid-19 model. Covid-19 infection duration of 14 days was considered. Sensitivity analyses for different morbidity scenarios, including post-acute consequences were presented.

Results: An estimated 70,421 people were infected (with and without symptoms) by Covid-19 in Malta (March 2020-1), out of which 1636 required hospitalisation and 331 deaths, contributing to 5478 DALYs. These DALYs positioned Covid-19 as the fourth leading cause of disease in Malta. Mortality contributed to 95\% of DALYs, while post-acute consequences contributed to $60 \%$ of morbidity.

Conclusions: Covid-19 over 1 year has impacted substantially the population health in Malta. Post-acute consequences are the leading morbidity factors that require urgent targeted action to ensure timely multidisciplinary care. It is recommended that DALY estimations in 2021 and beyond are calculated to assess the impact of vaccine roll-out and emergence of new variants.
\end{abstract}

Keywords: Burden of disease, Disability-adjusted life years, DALY, YLD, YLL, Population health, Malta, European burden of disease network, Coronavirus, Covid-19

\section{Background}

The outbreak of novel coronavirus SARS-CoV-2 has been a global pandemic since early 2020 [1]. The small European state of Malta reported its first Covid-19 case on the 7th of March 2020 [2]. The first Covid-19 wave was well controlled in Malta, with a relatively low mortality rate and a total cumulative positive cases of 108 per 100,000 [3]. The second Covid-19 wave initiated in mid-Summer and this saw a high mortality and admission rates to

\footnotetext{
*Correspondence: sarah.cuschieri@um.edu.mt

'Department of Anatomy, Faculty of Medicine and Surgery, University of Malta, Msida, Malta

Full list of author information is available at the end of the article
}

hospital $[4,5]$. The positivity and mortality rates were constantly high until mid-March 2021, where another instituted lockdown curbed the community spread [6]. Up till the end of March 2021, 6562 positive cases per 100,000 population have been reported with 88 deaths per 100,000 population [2].

Burden of disease assessments allow the estimation of a disease impact on a population by measuring the morbidity and mortality attribution and presenting it through a single metric called disability-adjusted life years (DALYs) [7]. Morbidity occurrence is translated through the estimation of years lived with disability (YLD) following the adjustment for the severity and the

(c) The Author(s). 2021 Open Access This article is licensed under a Creative Commons Attribution 4.0 International License, which permits use, sharing, adaptation, distribution and reproduction in any medium or format, as long as you give appropriate credit to the original author(s) and the source, provide a link to the Creative Commons licence, and indicate if changes were made. The images or other third party material in this article are included in the article's Creative Commons licence, unless indicated otherwise in a credit line to the material. If material is not included in the article's Creative Commons licence and your intended use is not permitted by statutory regulation or exceeds the permitted use, you will need to obtain permission directly from the copyright holder. To view a copy of this licence, visit http://creativecommons.org/licenses/by/4.0/ The Creative Commons Public Domain Dedication waiver (http://creativecommons.org/publicdomain/zero/1.0/) applies to the data made available in this article, unless otherwise stated in a credit line to the data. 
disability suffered due to a disease or injury. The mortality counts due to the disease or injury, are translated into estimates of years of life lost to premature mortality (YLL), using age-conditional life tables. DALYs are estimated by the combination of YLD and YLL [7]. This metric is a popular way used to assess the health impact at a population level and then translated as evidence to influence national policy makers.

The aim of this study was to estimate, for the first time, the direct impact of Covid-19 on the population of Malta for the first year (March 2020-2021). We also set to investigate the likely Covid-19 impact in relation to other causes of disease and injury, at a population level.

\section{Methods}

Data

Covid-19 mortality data between March 2020 and March 2021 were recorded from the daily Covid-19 bulletins issued by the Malta Ministry of Health [2]. However, mortality data did not differentiate from those dying with Covid-19 from those dying due to Covid-19, in line with WHO recommendations on assigning underlying cause of death [8]. Also, this group-level COVID-19 data did not provide details on the presence of any other underlying comorbidities among the infected individuals.

The Covid-19 positive cases were sub-divided according to the different health states, as shown in Table 1, based on the European Burden of Disease Network and the European Centre of Disease Prevention and Control (ECDC) Covid-19 consensus disease model [9]. Daily positive case numbers have been reported by the Malta Ministry of Health from the onset of the pandemic in Malta [2]. However, data on the different health states of the Covid-19 positive cases were provided on a weekly basis, every Friday, from the 7th of August 2020, following the initiation of the second wave in Malta [5]. Prior to this period, the Covid-19 situation was well controlled [3, 10]. Hence, weekly Covid-19 health states were not publicly provided except for the monthly intensive care unit (ICU - critical cases) admissions due to Covid-19 [11]. Therefore, estimations for the hospitalized Covid-19 cases (non-ICU, i.e., severe cases) from March to July 2020 were performed. An assumption was made that the proportion of Covid-19 admissions to hospital in August in relation to the total positive cases (not requiring ICU) was the same as that throughout March to July 2020. It was hence assumed that $1.3 \%$ of the Covid-19 cases not admitted to the ICU, required admission to a hospital ward i.e. were 'severe' cases.

Considering that in Malta a high swabbing rate and efficient contact tracing, up to secondary contact, has been in effect from the onset of the pandemic, it was noted that $20 \%$ of the reported positive cases (not requiring hospitalisation, i.e., moderate cases) were actually asymptomatic $[3,5,12]$. Hence, for this study's analyses, a reduction of $20 \%$ from the moderate cases was performed. However, the literature indicates that a proportion of Covid-19 will not be picked up through polymerase chain reaction (PCR) testing [13]. Indeed, the PCR sensitivity has been reported to be $97.2 \%$ [14]. Based on this, we have estimated that cases represent $80 \%$ of all infections, so have upscaled our estimates to reflect this to estimate the total number of asymptomatic and symptomatic infections. Additionally, it has been noted that approximately $20 \%$ of reported cases were actually asymptomatic $[3,5,12]$. To reflect this, we have also reduced the number of moderate cases by $20 \%$, since by virtue of requiring healthcare assistance, any severe or critical cases would be symptomatic. Therefore, we estimated that asymptomatic cases represented a total of $36 \%$ of all cases as follows: (i) $20 \%$ of all cases are asymptomatic and not reported (ii) $20 \%$ of the reported cases (80\%) are asymptomatic i.e. $16 \%$ of all cases.

\section{Analyses}

The modelling approach applied by this study to calculate the Covid-19 DALYs was based on the European Burden of Disease Network and the ECDC consensus disease model [9].

Table 1 Covid-19 health states, descriptions and disability weights [9]

\begin{tabular}{|c|c|c|}
\hline Health state & Description & $\begin{array}{l}\text { Disability weight ( } 95 \% \\
\text { uncertainty interval) }\end{array}$ \\
\hline Asymptomatic & Has infection but does not experience any symptoms & Nil \\
\hline $\begin{array}{l}\text { Moderate } \\
\text { (Community; seeking healthcare } \\
\text { assistance) }\end{array}$ & $\begin{array}{l}\text { Has symptoms and causing some difficulty with the daily activities. Does not } \\
\text { require hospital admission. }\end{array}$ & $0.051(0.032-0.074)$ \\
\hline $\begin{array}{l}\text { Severe (Hospitalised; non- } \\
\text { intensive care) }\end{array}$ & Has symptoms and causing great difficulty with daily activities. & $0.133(0.088-0.190)$ \\
\hline $\begin{array}{l}\text { Critical (Hospitalised; intensive } \\
\text { care) }\end{array}$ & Intensive care admission with or without respiratory support & $0.655(0.579-0.727)$ \\
\hline Post-acute consequences & $\begin{array}{l}\text { Person still feels symptoms such as tired, fatigue, pain all over body, persisting } \\
\text { after the acute infection }\end{array}$ & $0.219(0.148-0.308)$ \\
\hline
\end{tabular}


YLL was estimated by combining the death counts by five-year age-groups and sex. The estimates were calculated by multiplying the number of deaths in each agegroup by the age-conditional remaining life expectancy from the Global Burden of Disease (GBD) Study 2019 reference life table, where the same values are assigned to both males and females [15].

The symptomatic duration of 14 days was considered for each health state (critical, severe, moderate), following local and international reported durations of Covid-19 infection respectively $[16,17]$. Person-years were calculated as the sum of cases for each symptomatic health states (critical, severe, moderate), multiplied by 14, divided by 365.25 , reflecting the contribution of individual days to a complete year. This was carried out since DALYs use a year as the unit of time. An estimate of the post-acute consequence (long-Covid) YLD was calculated, where it was assumed that 13.3\% (one in seven) of the total Covid19 patients (symptomatic and asymptomatic) would suffer from long-Covid for a duration of 28 days [18]. The disability weights used for each health status was derived from the European Disability Weight Study (EDWS) and GBD 2019, as shown in Table $1[19,20]$.

YLD was estimated by multiplying person-years of infection with the disability weight for each health status (as per Table 1). The disability weight's uncertainty levels (UI) were also considered to measure the upper and lower limits of the YLD. DALYs were then calculated through the summation of YLL and YLD. The upper and lower limits of the DALYs were also calculated based on the UIs of the YLD.

Our estimate of Covid-19 DALYs was compared to the 2019 leading ranked causes of disease and injury for Malta, using estimates from GBD 2019 [21].

\section{Scenario analyses}

A number of uncertainties were present in our analyses especially when estimating YLD. The health state case numbers were not available prior to August 2020 (except for ICU on a monthly basis). When health state data was available, the cases were a snapshot of one particular day (Friday). Another uncertainty was the proportion of cases that suffered from post-acute Covid-19 consequences. Thus, in order to quantify the impact of these uncertainties, sensitivity analyses were performed. Sensitivity analyses were performed on the moderate cases and the post-acute consequences, since these were likely the most uncertain estimates. The sensitivity analyses assessed for the effect of an increase in moderate cases by 10,25 and $50 \%$ on the DALYs. We also assessed the effect of different transition probabilities from an acute to post-acute consequence have on the DALYs, when considering all Covid-19 cases and then symptomatic cases only. Finally, different scenarios were assumed with half or double the duration of post-acute consequences and different proportions of moderate cases, in order to minimise and maximise the YLD.

\section{Results}

Over the course of a year (March 2020 - March 2021), an estimated 70,421 people were infected by Covid-19 in Malta, both with and without symptoms. When considering the symptomatic cohort and taking into the account the duration of the Covid-19 infection; for an infection duration of 14 days, this reflected 1570 personyears. These generated a YLD of 93, as shown in Table 2 . Out of the three health states, the moderate symptomatic cohort translated the most to the symptomatic YLD. Post-acute consequences were estimated to have affected 9366 patients with a contributing YLD of 157, if symptoms persisted for 28 days. Out of the different health states, post-acute consequences had the highest impact on YLD while the severe cases had the lowest impact on YLD. The mortality for this time period was of 331 deaths, contributing to 5229 YLLs.

It was estimated that the DALYs due to Covid-19 were 5478. The highest contributor factor to the DALYs was mortality, with YLL representing 95\% of the DALYs with 16 YLL per Covid-19 death.

On comparing the DALYs for the top-ranking diseases and injuries as reported by GBD 2019 for Malta to this study's estimated Covid-19 DALYs, it was noted that the Covid-19 DALY ranked fourth, after ischemic heart disease (13,594 DALYs), low back pain (7106 DALYs) and diabetes (5796 DALYs), but before stroke (5096 DALYs).

\section{Scenario analyses}

A number of different sensitivity analyses, which varied assumptions on moderate cases (symptomatic community) and post-acute consequences health statuses were performed to evaluate their impact on DALYs, as shown in Table 3. Additionally, a combination of assumptions was performed to minimise and maximise the impact of YLD, with a range of 115 to 3606 (Table 3). The different defining scenarios involving the post-acute consequences and the combination assumption maximising the YLD, led to a higher estimated DALY than that estimated for Malta 2020. However, only when maximising the YLD, did the DALY represent a higher-ranking position for the cause of disease and disability for Malta, from initially placing fourth to second leading cause of disability.

\section{Discussion}

\section{Summary}

DALYs due to Covid-19 in Malta were likely to have had the fourth largest population health impact relative to other diseases and injuries for 2020. The majority of the 
Table 2 Measure of the population health impact of Covid-19

\begin{tabular}{|c|c|c|c|c|c|c|}
\hline \multirow[t]{2}{*}{ Health State } & \multicolumn{3}{|c|}{ Morbidity } & \multicolumn{2}{|c|}{ Mortality } & \multirow[t]{2}{*}{ DALYs (UI 95\%) } \\
\hline & Persons & Person - years & $\overline{\text { YLD (UI 95\%) }}$ & Deaths & YLL & \\
\hline Covid-19 infection & 70,421 & & $250(166-352)$ & 331 & 5229 & $5478(5395-5581)$ \\
\hline Symptomatic Covid-19 & & 1570 & $93(60-131)$ & & & \\
\hline Moderate & & 1507 & $77(48-112)$ & & & \\
\hline Severe & & 49 & $7(4-9)$ & & & \\
\hline Critical & & 14 & $9(8-10)$ & & & \\
\hline Post-acute consequences ${ }^{a}$ & 9366 & 718 & $157(106-221)$ & & & \\
\hline
\end{tabular}

duration of 28 days

population health loss was due to mortality as it represented 95\% of DALYs with 16 YLL per Covid-19 death. On comparing to the Scottish Covid-19 BoD study, our study's mortality contributed to a lesser percentage to the DALYs than Scotland, where the latter reported mortality to contribute to $98 \%$ of DALYs [22].

In our study, morbidity was driven mostly by postacute consequences, followed by symptomatic community cases (moderate). Indeed, when maximizing both post-acute consequences and moderate cases, the estimated DALY exceeded the impact of Covid-19 from fourth to the second leading cause of disability in Malta. Therefore, although Covid-19 individuals that require hospitalisation, with or without intensive care support, have a considerable ill-health impact, the post-acute consequences, also called "long-haulers" might pose the largest cumulative impact on the population health, although this follows a degree of uncertainty. This finding corresponds with the Scottish Covid-19 DALYs estimate although different time periods were considered in this study [23]. Nevertheless, it needs to be noted that following a comparison analyses between a Malta national burden of disease study for low back pain (LBP) to the GBD LBP estimates for Malta, it was concluded that the GBD overestimated the LPB burden [24]. Hence, it is likely that the Covid-19 DALYs estimated in this study may place at a higher-ranking position than fourth leading cause of disability in Malta.

Table 3 Covid-19 Morbidity sensitivity analyses with corresponding impact on YLD and DALYs, Malta, 2020

\begin{tabular}{|c|c|c|c|}
\hline Impacted health status & Sensitivity & YLD (UI 95\%) & DALYs (UI) \\
\hline \multirow[t]{3}{*}{ Moderate - Community cases } & Increase by $10 \%$ & $256(170-361)$ & $5484(5399-5589)$ \\
\hline & Increase by $25 \%$ & $267(178-377)$ & $5495(5406-5606)$ \\
\hline & Increase by $50 \%$ & $286(190-405)$ & $5514(5418-5633)$ \\
\hline \multirow[t]{4}{*}{ Post-acute consequences for all cases } & Transition probability of $26.6 \%$ & $406(272-571)$ & $5634(5501-5800)$ \\
\hline & Transition probability of $6.65 \%$ & $171(113-241)$ & $5399(5342-5470)$ \\
\hline & Duration of 56 days & $406(272-571)$ & $5634(5501-5800)$ \\
\hline & Duration of 14 days & $171(114-241)$ & $5399(5342-5470)$ \\
\hline \multirow{4}{*}{$\begin{array}{l}\text { Post-acute consequences for symptomatic cases } \\
\text { only }\end{array}$} & Transition probability of $26.6 \%$ & $275(184-388)$ & $5504(5413-5617)$ \\
\hline & Transition probability of $6.65 \%$ & $138(92-195)$ & $5367(5320-5424)$ \\
\hline & Duration of 56 days & $275(184-388)$ & $5504(5413-5617)$ \\
\hline & Duration of 14 days & $138(92-195)$ & $5367(5320-5424)$ \\
\hline \multirow[t]{4}{*}{ Combination to minimize the impact of YLD } & No increse in Moderate case & \multirow[t]{4}{*}{$115(76-163)$} & \multirow[t]{4}{*}{$5344(5305-5392)$} \\
\hline & $\begin{array}{l}\text { Transition to post-acute consequences for symptomatic } \\
\text { only }\end{array}$ & & \\
\hline & Half the transition of acute to post consequences (6.65\%) & & \\
\hline & Half the duration for post-acute consequences (14 days) & & \\
\hline \multirow[t]{4}{*}{ Combination to maximize the impact of YLD } & Increase Moderate cases by $50 \%$ & \multirow{4}{*}{$\begin{array}{l}3606(2295- \\
5201)\end{array}$} & \multirow{4}{*}{$\begin{array}{l}8834(7524- \\
10,429)\end{array}$} \\
\hline & Transition to post-acute for All cases & & \\
\hline & Double transition of acute to post consequences (26.6\%) & & \\
\hline & Double duration for post-acute consequences (56 days) & & \\
\hline
\end{tabular}




\section{Strengths and weaknesses}

The YLL calculation was based on the national reported cases (hospital, nursing homes and private residence deaths) that died while with Covid-19. Distinction was not made between those that died with Covid-19 or due to Covid-19. Since the onset of the pandemic, all deaths at hospital (even if initially negative for Covid-19), were tested for Covid-19 post-mortem however, deaths in private residents were not unless suspected by relatives that the deceased might have had Covid-19. This is more likely to have overestimated DALYs due to Covid-19, however this is counter-acted by the fact that deaths from early in the pandemic would have been less likely to have been identified as dying with Covid-19. The validity of the mortality estimate is furthermore supported by recent information on excess mortality. Compared to 2019, there have been 317 more deaths in 2020, which is in line with the number of reported Covid-19 deaths. Furthermore, caution needs to be put forward when comparing our study results to other Covid-19 burden of disease studies according to the mortality definition considered for the DALYs estimation.

The aspirational life table was used to calculate the YLL in order to facilitate comparative research considering that Covid-19 is a global issue, although acknowledge that this choice is an area of intense debate [25].

All admissions to hospital were tested for Covid-19, with PCR testing repeated if any symptoms develop during the patient's stay or the patient needed to undergo an invasive procedure. Hence, the Covid-19 pick up rate for in-patient cases was efficient. However, hospital data was only freely available on a weekly basis from August. Although assumptions had to be made for this time period, acute YLD is very small in relation to the YLL and so the impact of these assumptions will be minimal. However, one cannot dismiss the possibility that the assumptions might have led to overestimations or underestimations for some cases.

At a community level, a high swabbing rate has been implemented from the very start of the pandemic in Malta with contact tracing up to the second contact of an infected person [3]. Therefore, Covid-19 positive cases, whether symptomatic or asymptomatic, were picked up at an early stage of the disease. Based on the literature it was assumed that the total Covid-19 population consisted of an additional 20\% Covid-19 asymptomatic individuals that were never picked up [13]. However, this was an estimated proportion originating from the literature and does not necessarily reflect the local undetected Malta cases, hence we cannot exclude the possibility of overestimations or underestimations of this asymptomatic cohort.

The disease model to calculate the health loss due to Covid-19 morbidity was based on the European Burden of Disease Network and ECDC consensus method, which was adopted for both prevalence- or pathogenbased YLD calculations [9]. Meaning that any future presenting long-Covid conditions such as respiratory conditions, will be attributed to their relevant noncommunicable diseases through prevalence data.

It needs to be noted that comorbidity data for the Covid-19 positive cases was not available. Common comorbidities such as diabetes and cardiovascular disease have been reported to increase the risk of acquiring Covid-19 infection (morbidity) as well as have a higher chance of mortality due to Covid-19 [26, 27]. However, burden of disease studies, including the Global Burden of Disease (GBD) Study, perform adjustments to downscale YLD to correct for multimorbidity. Indeed, it is uncommon for the YLL to be adjusted in such cases [28]. In terms of our study, since the major contribution to the DALYs was from the YLL rather than YLD, any adjustments to reduce the YLD would not have influenced our findings.

In our calculations, the post-acute consequences were based on assumptions since long Covid-19 remains largely uncertain and different entities provide different definitions and durations [29, 30]. Indeed, the scenario analyses were performed to try out a number of different assumptions and scenarios, while evaluating the corresponding DALYs. However, since the YLD contributed to only $5 \%$ of the DALYs, fluctuations in post-acute consequences had a greater impact on DALYs only when maximizing the YLD health status outcome. It is recommended that further research is performed on postacute consequences to have a better understanding on the progress of this condition.

\section{Conclusions}

Covid-19 has bestowed a substantial burden upon Malta's population health. This study estimates the direct impact of Covid-19 in Malta and not the impact of lockdowns or implemented mitigation measures instituted throughout the duration of this pandemic. From our analyses it could be observed that Covid-19 presented as a novel disease in March 2020, however over 1 year it became the fourth leading cause of disability among the population. The burden of disease methodology used assessed for both the direct and indirect impacts of Covid-19. Indeed, it was clear that the largest morbidity impact of Covid-19 was arising from post-acute consequences. Although this is still an unknown territory, where even the definition is still debatable [29], it is clear that those that become 'long haulers' are attributing to higher DALY and inevitably will have a higher burden on the healthcare system. Indeed, more than 50 different symptoms have been reported to be experienced by these 'long haulers' with the commonest symptom being 
fatigue followed by headaches [31]. Therefore, it is recommended that further research on long Covid-19 is conducted at a national level. Additionally, long Covid19 should be considered as an urgent public health matter with engagement of a multi-disciplinary service to provide targeted healthcare service to this subpopulation.

Since the end of December 2020, Malta, has been administering Covid-19 vaccination to the population through a priority system [32]. Indeed, up to the time of writing (3rd May 2021), Malta has the fastest vaccination roll-out within the European Union (EU), with $25 \%$ of the total eligible population fully vaccinated and 54\% having had the first dose [2]. Covid-19 vaccination is considered as the tool to tackle Covid-19 infection. Hence it is recommended that DALYs estimations are measured periodically during 2021 and beyond to determine the vaccines' impact on the population, as well as determine the effect of emerging variants.

\section{Abbreviations}

Covid-19: Coronavirus Disease 2019; DALYs: Disability-Adjusted Life Years; ECDC: European Centre for Disease Prevention and Control; EDWS: European Disability Weight Study; GDB: Global Burden of Disease; ICU: Intensive Care Unit; YLD: Years Lived with Disability; YLL: Years of Life Lost to premature death

\section{Acknowledgements}

The authors would like to acknowledge the networking support from COST Action CA18218 (European Burden of Disease Network; https://www.burdeneu.net), supported by COST (European Cooperation in Science and Technology; https://www.cost.eu).

\section{Authors' contributions}

All authors contributed to the study design, writing, and reviewing of the article. SC was responsible for statistical analyses and interpretation of the data. All authors read and approved the final manuscript.

\section{Funding}

None received.

\section{Availability of data and materials}

The datasets used and/or analysed during the current study are all publicly available online on the Malta Ministry of Health website: https://www. facebook.com/sahhagovmt

\section{Declarations}

Ethics approval and consent to participate

Not applicable.

\section{Consent for publication}

Not applicable.

\section{Competing interests}

Authors declare no competing interests.

\section{Author details}

${ }^{1}$ Department of Anatomy, Faculty of Medicine and Surgery, University of Malta, Msida, Malta. ${ }^{2}$ Department of Public Health, Faculty of Medicine and Surgery, University of Malta, Msida, Malta. ${ }^{3}$ Directorate for Health Information and Research, Ministry for Health, Gwardamangia, Malta. ${ }^{4}$ Department of Epidemiology and Public Health, Sciensano, Brussels, Belgium. ${ }^{5}$ Department of Veterinary Public Health and Food Safety, Ghent University, Merelbeke,
Belgium. ${ }^{6}$ Public Health Adviser, Place and Wellbeing Directorate, Public Health Scotland, Glasgow, Scotland.

Received: 2 July 2021 Accepted: 28 September 2021

Published online: 09 October 2021

\section{References}

1. World Health Orgainzation. WHO Director-General's opening remarks at the Mission briefing on COVID-19. Geneva: World Health Organization; 2020 Cited 2020 Sep 11. Available from: https://www.who.int/dg/speeches/detail/ who-director-general-s-opening-remarks-at-the-mission-briefing-on-covid-1 9-12-march-2020

2. COVID-19 Public Health Response Team - Ministry for Health. COVID-19 Data Management System. 2021. Available from: https:// deputyprimeminister.gov.mt/en/health-promotion/covid-19/Pages/covid-19infographics.aspx

3. Cuschieri S. COVID-19 panic, solidarity and equity - the Malta exemplary experience. J Public Health. 2020;30:1-6. Cited 2020 Jun 3. Available from https://doi.org/10.1007/s10389-020-01308-w.

4. Cuschieri S, Pallari E, Hatziyianni A, Sigurvinsdottir R, Sigfusdottir ID, Sigurðardóttir ÁK. Mortality comparisons of COVID-19 with all-cause and non-communicable diseases in Cyprus, Iceland and Malta: lessons learned and forward planning. Public Health. 2021; Cited 2021 May 3; Available from: https://www.sciencedirect.com/science/article/pii/S0033350621001359.

5. Cuschieri S, Balzan M, Gauci C, Aguis S, Grech V. Mass Events Trigger Malta's Second Peak After Initial Successful Pandemic Suppression. J Commun Health. 2020:1-8. Springer. Cited 2020 Sep 17. Available from. https://doi. org/10.1007/s10900-020-00925-6.

6. Times of Malta. Malta enters month-long shutdown as schools, services close, amid virus spike: Times of Malta; 2021. Cited 2021 Mar 18. Available from: https://timesofmalta.com/articles/view/watch-prime-minister-a nnouncing-new-covid-19-measures.857200

7. Murray CJL, Lopez AD. The Global Burden of Disease: A Comprehensive Assessment of Mortality and Disability from Diseases, Injuries and Risk Factors in 1990 and Projected to 2020. Boston: Harvard School of Public Health; 1996.

8. World Health Organization. Cause of Death COVID-19. 2020. Available from: https://www.who.int/classifications/icd/Guidelines_Cause_of_Death_ COVID-19.pdf?ua=1

9. Wyper GMA, Assunção RMA, Colzani E, Grant I, Haagsma JA, Lagerweij G, et al. Burden of Disease Methods: A Guide to Calculate COVID-19 DisabilityAdjusted Life Years. Int J Public Health. 2021;66:4 Cited 2021 Apr 30 Available from: https://www.ssph-journal.org/articles/10.3389/ijph.2021.61 9011/full.

10. Cuschieri S. COVID-19: the transition towards a new normal-experiences from the European country of Malta. J Public Health (Bangkok). 2021:1-8. Cited 2021 Mar 7. Available from. https://doi.org/10.1007/s10389-021-01486-1.

11. Arena J. Two out of every five COVID patients who needed a ventilator died. 2021 Times of Malta. Cited 2021 Apr 30. Available from: https:// timesofmalta.com/articles/view/two-out-of-every-five-covid-patients-whoneeded-a-ventilator-died.845984

12. Caruana C. 20 per cent of August's COVID-19 patients have no symptoms. 2020 Times of Malta. Cited 2020 Aug 22. Available from: https://timesofma Ita.com/articles/view/80-per-cent-of-augusts-covid-19-patients-have-nosymptoms. 812883

13. Oran DP, Topol EJ. The Proportion of SARS-CoV-2 Infections That Are Asymptomatic : A Systematic Review. Ann Intern Med. 2021. Cited 2021 Apr 26. Available from. https://doi.org/10.7326/M20-6976.

14. Böger B, Fachi MM, Vilhena RO, Cobre AF, Tonin FS, Pontarolo R. Systematic review with meta-analysis of the accuracy of diagnostic tests for COVID-19. Am J Infect Control. 2021:49(1):21-9 Cited 2021 Sep 9. Available from: http://www.ncbi.n/m.nih.gov/pubmed/32659413.

15. Institute for Health Metrics and Evaluation (IHME). Reference Life Table GHDx. Global Burden of Disease Study 2019 (GBD 2019). 2019 Cited 2021 Apr 30. Available from: http://ghdx.healthdata.org/record/ihme-data/globalburden-disease-study-2019-gbd-2019-reference-life-table

16. Centers for Disease Control and Prevention. Interim Clinical Guidance for Management of Patients with Confirmed Coronavirus Disease (COVID-19). Clinical Care Guidance. 2021

17. Costa M. 16 days: the average recovery time for COVID-19 patients in Malta. Malta today . 2020 Cited 2021 Apr 30. Available from: https://www.malta 
today.com.mt/news/national/102573/16_days_the_average_recovery_time_ for_covid19_patients in malta\#t.YlurhyORogp

18. Sudre CH, Murray B, Varsavsky T, Graham MS, Penfold RS, Bowyer RC, et al. Attributes and predictors of long COVD. Nat Med. 2021;27(4):626-31 Cited 2021 Apr 30. Available from: httpj//wnw.nature.com/articles/s41591-021-01292-y.

19. Haagsma JA, Maertens de Noordhout C, Polinder S, Vos T, Havelaar AH, Cassini A, et al. Assessing disability weights based on the responses of 30,660 people from four European countries. Popul Health Metr. 2015;13(1): 10 Cited 2021 Apr 30. Available from. https://doi.org/10.1186/s12963-0150042-4.

20. Institute for Health Metrics and Evaluation (IHME). Institute for Health Metrics and Evaluation (IHME). 2019. Available from: http://ghdx.healthdata. org/.

21. Institute for Health Metrics and Evaluation (IHME). Malta GBD Compare | IHME Viz Hub. 2019 GBD CompareCited 2021 Apr 30. Available from: https://vizhub.healthdata.org/gbd-compare/\#.

22. GMA W, Fletcher $\mathrm{E}$, Grant I, Harding O, de Haro MMTSD, et al. Inequalities in population health loss by multiple deprivation: COVID-19 and pre-pandemic all-cause disability-adjusted life years (DALYS) in Scotland. Int J Equity Health. 2021; 20(1):214. Available from: https://doi.org/10.1186/s12939-02101547-7.

23. Wyper G, Fletcher E, Grant I, McCartney G, Fischbacher C, Harding O, et al. Measuring the direct population impact of COVID-19 in Scotland, 2020: estimating disability-adjusted life years (DALYs) during the first full calendar year. 2021 SocArXiv ey36d, Cent Open Sci. Cited 2021 Apr 30; Available from: https://ideas.repec.org/p/osf/socary/ey36d.htm

24. Cuschieri S, Wyper GMA, Calleja N, Gorasso V, Devleesschauwer B. Measuring disability-adjusted life years (DALYs) due to low back pain in Malta. Arch Public Health. 2020;78:68 Cited 2021 Apr 29. Available from: http://www.ncbi.nlm.nih.gov/pubmed/32760586.

25. Devleesschauwer B, McDonald SA, Speybroeck N, GMA W. Valuing the years of life lost due to COVID-19: the differences and pitfalls. Int J Public Health. 2020;65(6):719-20 Cited 2021 May 12. Available from. https://doi.org/10.1 007/500038-020-01430-2.

26. Wang $B$, Li R, Lu Z, Huang Y. Does comorbidity increase the risk of patients with COVID-19: evidence from meta-analysis. Aging (Albany NY). 2020;12(7): 6049-57 Cited 2021 Sep 9. Available from: http://www.ncbi.nlm.nih.gov/ pubmed/32267833.

27. Ssentongo P, Ssentongo AE, Heilbrunn ES, Ba DM, Chinchilli VM. Association of cardiovascular disease and 10 other pre-existing comorbidities with COVID-19 mortality: A systematic review and meta-analysis. Hirst JA. PLoS One. 2020 ;15(8):e0238215 Cited 2021 Sep 9. Available from: https://doi. org/10.1371/journal.pone.0238215

28. HBM H, MHD P, BEP S, Boshuizen HC, MJJC P, van Gool CH. Accounting for multimorbidity can affect the estimation of the Burden of Disease: a comparison of approaches. Arch Public Heal. 2016;74(1):37 Cited 2021 Sep 9. Available from. https://doi.org/10.1186/s13690-016-0147-7.

29. Fernández-de-Las-Peñas C, Palacios-Ceña D, Gómez-Mayordomo V, Cuadrado ML, Florencio LL. Defining Post-COVID Symptoms (Post-Acute COVID, Long COVID, Persistent Post-COVID): An Integrative Classification. Int J Environ Res Public Health. 2021;18(5) Cited 2021 Apr 29. Available from: http://www.ncbi.nlm.nih.gov/pubmed/33807869.

30. Huang Y, Pinto MD, Borelli JL, Mehrabadi MA, Abrihim H, Dutt N, et al. COVID Symptoms, Symptom Clusters, and Predictors for Becoming a LongHauler: Looking for Clarity in the Haze of the Pandemic. medRxiv Prepr Serv Heal Sci. 2021; Cited 2021 Apr 24; Available from: http://www.ncbi.nlm.nih. gov/pubmed/33688670.

31. Lopez-Leon S, Wegman-Ostrosky T, Perelman C, Sepulveda R, Rebolledo PA, Cuapio A, et al. More than 50 long-term effects of COVID-19: a systematic review and meta-analysis. Sci Rep. 2021;11(1):16144 Cited 2021 Sep 9. Available from: https://www.nature.com/articles/s41598-021-95565-8.

32. Ministry of Health Malta. Vaccines: Ministry of Health Malta; 2021. Available from: https://deputyprimeminister.gov.mt/en/health-promotion/covid-19/Pa ges/vaccines.aspx

\section{Publisher's Note}

Springer Nature remains neutral with regard to jurisdictional claims in published maps and institutional affiliations.

\section{Ready to submit your research? Choose BMC and benefit from:}

- fast, convenient online submission

- thorough peer review by experienced researchers in your field

- rapid publication on acceptance

- support for research data, including large and complex data types

- gold Open Access which fosters wider collaboration and increased citations

- maximum visibility for your research: over $100 \mathrm{M}$ website views per year

At BMC, research is always in progress.

Learn more biomedcentral.com/submissions 